\title{
Esquema de Controle de Admissão de Fluxos Multifractais de Tráfego de Redes através de Estimação de Probabilidade de Perda
}

\author{
Jeferson Wilian de Godoy Stênico, Flávio Henrique Teles Vieira e Lee Luan Ling
}

\begin{abstract}
Resumo--Neste artigo, propomos um esquema de Controle de Admissão para fluxos de tráfego de redes, considerando características multifractais. Inicialmente, fazemos uma breve descrição das propriedades do tráfego multifractal de redes. Em seguida, descrevemos alguns algoritmos existentes relacionados a mecanismos de controle de admissão de conexão. Por fim, comparamos o desempenho do esquema de controle de admissão proposto com outras abordagens utilizando várias séries reais de tráfego com diferentes características estatísticas, principalmente propriedades multifractais. Avaliamos também, qual o impacto de uma modelagem mais precisa no controle de admissão de fluxos em uma rede de comunicações.
\end{abstract}

Palavras-Chaves- Análise de Tráfego, Processos Multifractais, Controle de Admissão.

Abstract--In this paper, we propose an admission control scheme for network traffic flows with multifractal characteristics. Initially we briefly introduce some properties of multifractal networks traffic and describe some existing algorithms which implement the related admission control mechanisms. Then, we compare the performance of the proposed admission control scheme with other approaches using real-traffic series with different statistical characteristics, especially those with multifractal properties. To conclude, we evaluate some advantages of using multifracal based traffic modeling in terms of quality of flow admission control in communication networks.

Index Terms-Traffic analysis, Multifractal Processes, Admission Control.

\section{INTRODUÇÃO}

Atualmente as redes de comunicações devem lidar com um considerável número de tipos de tráfegos distintos, compartilhando recursos comuns através da multiplexação estatística. A eficiência no compartilhamento destes recursos de rede depende das características estatísticas do tráfego.

Análises de várias medidas de tráfego mostraram que uma grande variedade de fluxos de tráfego, tanto em redes WAN quanto em LANs exibem um comportamento auto-similar, e a maioria dos esforços se concentraram na modelagem da dependência de longo prazo (LRD) destes processos [7].

Jeferson Wilian de Godoy Stênico e Lee Luan Ling, Departamento de Comunicação da Faculdade Engenharia Elétrica e Computação ,Unicamp,

Flávio Henrique Teles Viera, Escola de Engenharia Elétrica e Computação -UFG

E-mails: \{jstenico,lee\}@decom.fee.unicamp.br,flavio@eeec.ufg.br
Tráfegos auto-similares apresentam comportamento invariante à escala, exibindo similaridades estruturais através de uma gama de escalas de tempo (segundos, minutos, horas). A auto-similaridade presente nos traços de tráfego pode ser caracterizada pelo parâmetro de Hurst. Uma descrição completa do tráfego de redes requer o conhecimento de sua natureza dinâmica, não apenas sobre escalas de tempo grandes, mas também em escalas de tempo pequenas (milisegundos). Neste sentido, se incluem abordagens que se baseiam não só na captura da dependência de longo prazo, mas também das propriedades multifractais, que estão relacionadas com a alta variabilidade de tráfego encontrada em traços de tráfego principalmente em escalas pequenas de tempo.

Para alguns modelos monofractais simples tais como o $\mathrm{fBm}$ (fractional Brownian motion), o expoente que governa o comportamento de singularidade local é identicamente igual ao parâmetro de Hurst relacionado com a dependência de longo prazo do tráfego de redes. Entretanto, para processos gerais representativos de tráfego de redes de dados, o comportamento local do tráfego depende do tempo e é mais bem descrito através do espectro multifractal dos expoentes de singularidade. Assim, a análise multifractal pode prover novos conhecimentos para que se ajustem modelos para os dados reais de tráfego de redes.

O comportamento observado em escalas de tempo menores é devido principalmente aos protocolos e mecanismos de controle de congestionamento fim-a-fim, como por exemplo, o controle de fluxo TCP, que regulam as interações complexas entre diferentes conexões em uma rede [1].

As redes IP têm se tornado cada vez mais uma plataforma padrão de suporte às aplicações com demandas de qualidade de serviços distintas, sendo o controle de admissão de conexão um importante mecanismo de gerenciamento de tráfego para este fim.

Utilizam-se algoritmos de controle de conexão para determinar na chegada de um fluxo, se a nova conexão é aceita ou não. A utilização da rede e requisitos de qualidade de serviço (QoS) são fatores que necessitam ser considerados nas decisões de admissão. Um algoritmo eficiente de controle de admissão busca alcançar alta utilização da rede, enquanto provê os requisitos de qualidade de serviço para todas as conexões aceitas.

A disponibilidade de um mecanismo de controle de admissão eficiente e otimizado será um fator fundamental no desempenho das redes modernas com e sem fio. É importante 
ressaltar que esse processo não é trivial, principalmente devido às mudanças no comportamento do tráfego. A fim de verificarmos o impacto de uma modelagem multifractal para o tráfego de redes, apresentaremos neste trabalho um algoritmo de controle de admissão para fluxos de tráfego com características multifractais.

O artigo está organizado da seguinte forma: na seção II, faremos uma descrição das propriedades multifractais do tráfego de redes. Na seção III discursamos sobre mecanismos de controle de admissão de conexões, fazendo uma breve explanação de alguns algoritmos já existentes. Na seção IV, introduzimos uma nova proposta para o processo de controle de admissão baseada em estimação de probabilidade de perda para tráfego multifractal. Na seção V mostramos os resultados obtidos para diferentes tipos de traços de tráfego. Na seção VI apresentamos as conclusões obtidas.

\section{CARACTERIZAÇÃO DE TRÁFEGO MULTIFRACTAL}

Sinais multifractais são sinais singulares em quase todos os pontos e foram originalmente estudados como objetos degenerados de puro interesse matemático. Benoit Mandelbrot foi o primeiro a reconhecer que este tipo de fenômeno é encontrado em todo lugar, como por exemplo, registros econômicos tais como os índices de bolsas de valores, dados fisiológicos tais como as batidas do coração, etc [23].

Após a constatação das características fractais do tráfego de dados, pesquisadores da área, argumentavam que, ao contrário do que vinha acontecendo, as características locais das fontes de tráfego, não deveriam ser ignoradas. A questão era determinar onde o impacto dessas características é mais acentuado na rede. Foi demonstrado que o impacto destas características apresentam-se quando se analisa o tráfego de redes em escalas menores de tempo (centenas de milisegundos e menos), e que as características empíricas observadas no tráfego são consistentes com multifractais. Estas características multifractais sugerem uma classe de modelos que provêem uma mais completa e apurada descrição de tráfego real se comparado com modelos anteriores.

No contexto de redes, multifractais estão estendendo e refinando de uma forma natural o comportamento fractal ou auto-similar observado em tráfego de dados medidos. De fato, a auto-similaridade, é caracterizada por uma única lei de comportamento em escala que se mantém globalmente no tempo e essencialmente envolve apenas um parâmetro, o parâmetro de Hurst. Por outro lado, modelos multifractais permitem leis de comportamento em escala dependentes do tempo, e assim, oferecem maior flexibilidade em descrever os fenômenos irregulares que são encontrados no tempo. Estes fenômenos são tipicamente causados por mecanismos de rede específicos, que operam em escalas de tempo pequenas e, dependendo do estado da rede podem ter impactos mais ou menos severos na dinâmica de pacotes em uma conexão.

Neste trabalho, adotamos um modelo multifractal para o tráfego a fim de calcularmos a probabilidade de perda em um enlace. Para isso, primeiramente propomos uma aproximação da variância do tráfego em função do tempo e derivamos uma equação para a probabilidade de perda de dados em um enlace
[22]. Por meio desta equação proposta, apresentamos um esquema de controle de admissão, onde se considera as características multifractais do tráfego de redes.

\section{MeCAnismos de CONTROLE DE AdMISSÃo DE CONEXÃO}

Algoritmos de controle de admissão (CAC - Connection Admission Control) podem ser classificados como determinísticos ou estatísticos. Esquemas determinísticos tipicamente requerem apenas simples parâmetros de tráfego, definidos através da sinalização da conexão, tais como taxa de pico ou taxa média. Isto simplifica o processo de decisão, porém, as decisões tomadas podem tornar-se pouco precisas. Por outro lado, esquemas estatísticos tipicamente necessitam de mais parâmetros de tráfego e um modelo de tráfego explícito. Esquemas estatísticos freqüentemente consideram ganhos de multiplexação, fato este que os fazem atrativos, principalmente quando os tráfegos apresentam surtos.

Vários algoritmos de controle de conexão foram propostos na literatura [8],[9],[10],[11]. Estes algoritmos tomam decisões de controle de conexão baseado em suas respectivas concepções sobre características de tráfego, requisitos de qualidade de serviço e utilização da rede.

Os mecanismos de controle de admissão de conexão possuem três componentes básicos: descritores de tráfego, critérios de admissão e processos de medida de tráfego.

Descritores de tráfego são um conjunto de parâmetros que caracterizam as fontes de tráfego. Quando o tráfego apresenta grande intensidade de surtos, a utilização da rede pode tornarse muito baixa caso o controle de admissão esteja baseado apenas em parâmetros providos no estabelecimento da conexão. Assim, para se obter maiores taxas de utilização, o controle de admissão deve monitorar a dinâmica da rede e utilizar medidas tais como carga instantânea da rede e atraso de pacotes para fazer suas decisões de admissão. Esquemas baseados em medidas são chamados controle de admissão baseados em medida (MBAC)[24].

Em redes MBAC, um processo de medida é uma entidade lógica que realiza medidas da dinâmica dos dados da rede e fornece as informações obtidas aos algoritmos de controle de admissão. Isso faz com que esquemas MBAC se beneficiem da multiplexação estatística do tráfego para maximizar a utilização da rede.

Os critérios de admissão são as regras pelas quais os esquemas de controle de admissão aceitam ou rejeitam um fluxo. Assim, uma decisão de controle de admissão é freqüentemente feita baseada em uma estimação do efeito que o novo fluxo terá em outros fluxos e a utilização da rede em questão.

Como se pode observar em [2], vários métodos de controle de admissão são apresentados, dentre eles resumidamente temos os seguintes:

\section{A. Baseado em taxa média e taxa de pico}

Nesta classe, a fonte de tráfego é caracterizada por sua taxa de pico e ou por sua taxa média. Um dos principais exemplos de controle de admissão desta classe é apresentado em [26]. Neste artigo, os autores consideram um modelo On-Off para as fontes de tráfego, estimando a probabilidade de perda para 
o multiplexador sem buffer. Esta probabilidade de perda é então usada em um esquema de controle de admissão, indicando se os fluxos de tráfego podem ser admitidos no multiplexador.

\section{B. Baseado em Probabilidade de Perda Virtual:}

Consideraremos uma fonte $j[16]$, caracterizada pela taxa média $(A V G)$, e a taxa de pico $(M A X)$. Para uma fonte de vídeo, por exemplo, existem períodos ativos e períodos ociosos, desta forma a probabilidade da fonte estar ativa é dada por ( $A V G / M A X)$ e a probabilidade de estar em um estado ocioso, é dado por 1 - (AVG/MAX) e a taxa de tráfego será constante se, $A V G$ for igual a $M A X$.

Usando essa taxa de distribuição, um algoritmo de controle de admissão é designado para aproximar a probabilidade de perda em um multiplexador. Com isso, a qualidade de serviço (QoS) é medida em termos da probabilidade de perda virtual . A probabilidade de perda ocorre se e somente se, a taxa de pico agregado exceder a capacidade do servidor.

Para este método de controle de admissão é derivado apenas pelos paramentos $M A X$ e $A V G$ do tráfego [3],[4],[6], onde, a taxa de pico agregado para uma carga com $n$ fontes ativas é definido como, n.MAX.

A probabilidade de perda virtual é dada, da razão do excesso de trafego $O F$, pela utilização do trafego $(\rho)$. Denotamos o número de fontes multiplexada em um link por $N$.

Com isso temos as seguintes equações:

$$
\begin{gathered}
p v=O F / \rho \\
O F=\sum_{(n \cdot M A X-C) \geq 0}^{n=N} p(n)(n \cdot M A X-C) \\
\rho=N \cdot A V G
\end{gathered}
$$

onde $p(n)$ é a probabilidade que $n$ de $N$ fontes esteja ativa, isto é:

$$
p(n)=\left(\begin{array}{l}
N \\
n
\end{array}\right)\left(\frac{A V G}{M A X}\right)^{n}\left(1-\frac{A V G}{M A X}\right)^{N-n}
$$

Para este modelo, quando a taxa de pico agregada for menor que a capacidade do servidor, se assume que não ocorre perda. Mesmo que a condição seja válida, devido a um curto prazo de carga flutuante causada por chegadas simultâneas de diferentes tipos de fontes, a carga de tráfego excede instantaneamente a capacidade do servidor. E por essa razão, o buffer deve ter uma capacidade apropriada em um sistema real para prevenir a perda devido ao curto prazo de carga flutuante. É necessário um buffer com capacidade derivado de uma fila M/D/1 [8],[13].

\section{Baseado em banda efetiva aditiva}

Vários algoritmos de controle de admissão baseados em banda efetiva foram propostos na literatura, usando Teoria dos Grandes Desvios [18], decomposição em valores singulares de fluxos Markovianos [19] e Cálculo de Rede [20]. Para acomodar conexões de taxa variáveis de modo a atender seus requisitos de QoS descritos por suas bandas efetivas. Neste caso, a seguinte condição deve ser satisfeita [21]:

$$
\sum_{i=1}^{n} n_{i} c_{i} \leq C
$$

onde $C$ é a capacidade do enlace, $c_{i}$ é a banda efetiva para conexões do tipo $i$, e $n$ é o número de conexões do tipo $i$.

\section{Algoritmo Baseado na Máxima Variância (MVA)}

Sejam $A_{j}[s, t]$, o fluxo $j$ de tráfego de chegada no intervalo $[s, t], X_{t}$ como:

$$
X_{t}=\sum_{j} A_{j}[s-t, s]-C t
$$

Segundo os autores em [17], a Probabilidade de Perda de dados (pacotes, bytes, etc) é dada por:

$$
P(Q>B)=P\left(\operatorname{SupX}_{t}>B\right)
$$

A abordagem MVA se baseia na observação de que, se $X_{t}$ é Gaussiano, limitantes exatos e aproximações para o lado direito da equação (7) podem ser obtidos. Como $X_{t}$ corresponde à agregação de chegadas de um grande número de fontes, pelo Teorema do Limite Central, a hipótese de $X_{t}$ ser gaussiano é razoável em redes de alta velocidade. Para $X_{t}$ Gaussiano a Máxima variância (MVA) normalizada, é dada por:

$$
\sigma_{B}^{2}=\frac{\operatorname{var}\left\{X_{t}\right\}}{\left(B-E\left(X_{t}\right)\right)^{2}}
$$

Sendo $X_{t}$ Gaussiano, pode-se dizer que o instante de tempo $t$ em que a variância normalizada $\sigma_{B}^{2}$ for máxima é o mesmo instante em que a probabilidade de perda $P\left(X_{t}>B\right)$ atinge seu valor máximo, onde $B$ é o tamanho do buffer. Dessa forma, um fluxo de tráfego é aceito se a estimativa da probabilidade de perda $P\left(X_{t}>B\right)$ não ultrapassar um determinado valor [5]. Assim, uma aproximação utilizada para estimar a equação (7) é:

$$
P\left(\operatorname{SupX}_{t}>B\right) \approx \max _{t} P\left(X_{t}>B\right)
$$

\section{MÉtodo de Controle de Admissão Proposto}

Uma vez que a estimação de parâmetros multifractais dos fluxos de tráfego requer algoritmos relativamente complexos, desenvolver um algoritmo de controle admissão que leve em consideração essas características pode resultar em algoritmos com elevada complexidade computacional. Nesta seção propomos uma estratégia para o controle de admissão a partir da suposição de que a variância dos traços de tráfego tem um decaimento exponencial em função do tempo e dos expoentes de Hölder. Baseado em [22] vemos que, processos multifractais dependem fortemente dos momentos de primeira e segunda ordem para um processo multifractal, que são dados por [15]:

$$
\begin{gathered}
\mu=\lambda T \\
\sigma^{2} \sim \tau^{2 \alpha(T)}
\end{gathered}
$$

Observando a equação (11) temos que a variância (momento de segunda ordem) de processos multifractais se relacionam com o expoente de Holder $\alpha(T)$.

Dessa forma considerando $X(t)$ um processo multifractal com distribuição log-normal dada por: 


$$
f_{X(t)}(x)=\frac{1}{x \theta \sqrt{2 \pi}} e^{\frac{-(\ln (x)-\omega)^{2}}{2 \theta^{2}}}
$$

onde $\omega$ e $\theta$ são encontrados através da média e da variância do processo $X(t)$ dados por:

$$
\begin{gathered}
\omega=\ln \mu-\frac{1}{2} \ln \left(\frac{\sigma^{2}}{\mu^{2}}+1\right) \\
\theta=\sqrt{\ln \left(\frac{\sigma^{2}}{\mu^{2}}+1\right)}
\end{gathered}
$$

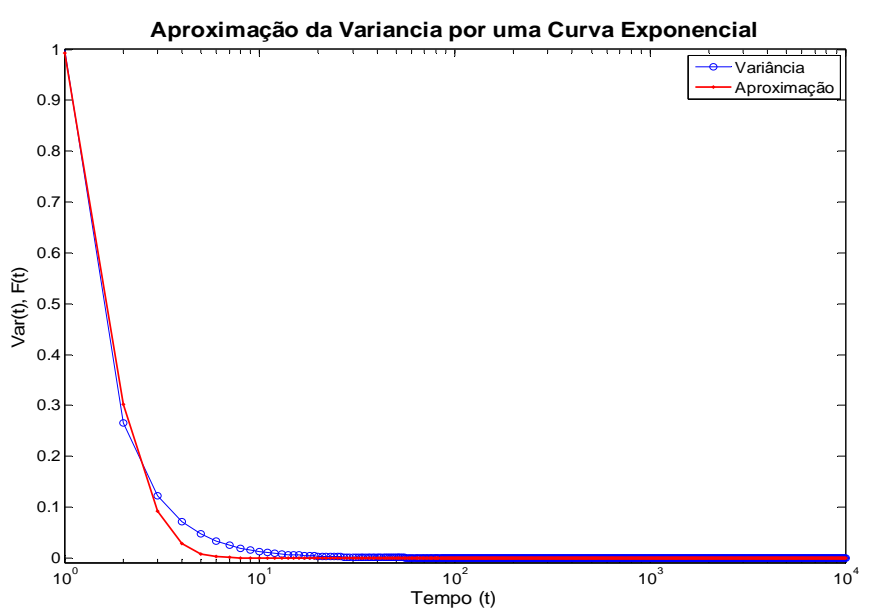

Fig 1 Variância versus Tempo e Função Exponencial Utilizada para o Traço de Tráfego dec-pkt-3

Para os traços de tráfego utilizados, constatou-se que é possível utilizar a função exponencial $f(T)=a \exp (b T)$ para caracterizar a variância em termos do tempo e do expoente de Hölder, conforme pode ser visto na Figura 1. Assumindo um comportamento exponencial para a variância e substituindo a equação (10) em (13) e (14) temos:

$$
\begin{array}{r}
\omega=\ln \left[\frac{\lambda T}{\sqrt{\left(c / \lambda^{2}\right) a \exp (b T)+1}}\right] \\
\theta=\sqrt{\left(c / \lambda^{2}\right) a \exp (b T)+1}
\end{array}
$$

onde $c$ é uma constante

Baseando-se na teoria de Benes [12] e como um processo multifractal apresenta distribuição lognormal, a probabilidade de perda em regime permanente para processos multifractais em um fila pode ser dada por:

$$
P_{\text {steady }}=\left.\left(1-\frac{\lambda}{C}\right) \int_{0}^{\infty} \frac{1}{x \theta \sqrt{2 \pi}} e^{\frac{-(\ln (x)-\omega)^{2}}{2 \theta^{2}}}\right|_{x=C u+q} d u
$$

Substituindo as equações (15) e (16) em (17) obtemos a seguinte equação para a probabilidade de perda:

$$
P_{\text {steady }}=\left(1-\frac{\lambda}{C}\right) \int_{0}^{\infty} \frac{\exp \left[\frac{-\left[\ln \left((C T+q) \sqrt{\left(c / \lambda^{2}\right) a \exp (b T)+1}\right)-\ln (\lambda T)\right]^{2}}{2 \ln \left(\left(c / \lambda^{2}\right) a \exp (b T)+1\right)}\right]}{\sqrt{2 \pi \ln \left(\left(c / \lambda^{2}\right) a \exp (b T)+1\right)}(C T+q)} d T
$$

Dessa forma, um fluxo de tráfego será aceito se a estimativa da probabilidade de perda $P_{\text {steady }}$ não ultrapasse um determinado limiar.

O método de controle de admissão proposto consiste basicamente de se calcular a probabilidade de perda de dados através da equação (18) a medida que se pretende estabelecer uma nova conexão. Ou seja, no método proposto, é necessário que para cada nova conexão, sejam calculados alguns parâmetros, dentre eles a média $(\lambda)$ do fluxo de tráfego, os coeficientes $a$ e $b$ da função exponencial usada na aproximação da variância, obtidos através do método de mínimos quadrados, além de estabelecer os valores para o servidor a ser utilizado, (Capacidade $(C)$ e Tamanho do buffer $(q))$. Assim, uma conexão é aceita caso o valor da probabilidade de perda dada pela equação (18) esteja abaixo do desejado.

\section{TESTES EXPERIMENTAIS}

Utilizamos nas simulações diferentes tipos de traços de tráfegos, tais como tráfegos TCP/IP, tráfegos de vídeos, tráfegos sintéticos, apresentando características monofractais e características multifractais.

Dividiremos nossa análise em vários experimentos de acordo com o tipo de tráfego que é considerado. Para o primeiro experimento foi usado apenas o traço de tráfego TCP/IP, dec-pkt-2 [27] na escala de 10ms, com 18563 amostras. Esse traço de tráfego tem um comportamento que tende a ser monofractal [28].

Para o segundo experimento foram utilizados vários traços de tráfegos TCP/IP monofractais e multifractais, dec-pkt-2100, dec-pkt-3-100, dec-pkt-3-512, lbl-tcp-3, lbl-pkt-4-100, lbl-pkt-5-100, lbl-pkt-4-512, lbl-pkt-5-512, BC-pAug89-200, BC-pAug89-400, todos com 7000 amostras.

Os experimentos 3 e 4 foram utilizados traços sintéticos multifractais gerados pelo FRACLAB segundo o modelo mBm [14]. As séries geradas possuem 16384 amostras.

Já para o experimento 5 utilizamos traços de tráfegos de vídeos retirados de [13], cada série apresenta 89998 amostras.

Para a realização das simulações consideramos as seguintes configurações para o servidor de rede.

TABELA I CONFIGURAÇÃO DO SISTEMA

\begin{tabular}{|c|c|c|}
\hline $\begin{array}{c}\text { Série de } \\
\text { Tráfego }\end{array}$ & $\begin{array}{c}\text { Capacidade do } \\
\text { Servidor }\end{array}$ & $\begin{array}{c}\text { Tamanho de } \\
\text { Buffer }\end{array}$ \\
\hline $\begin{array}{c}\text { TCP/IP } \\
\text { (DEC210ms) }\end{array}$ & $1.6 e+004$ & $4 e+004$ \\
\hline $\begin{array}{c}\text { TCP/IP (Varias } \\
\text { series) }\end{array}$ & $5 e+004$ & $7 e+005$ \\
\hline Multifractal & $1 e+004$ & $4 e+004$ \\
\hline fBm & $2 e+004$ & $4 e+004$ \\
\hline Vídeo & $3 e+004$ & $4 e+004$ \\
\hline
\end{tabular}

As Figuras 1, 2, 3, 4, 5 são referentes as seguintes séries de tráfego respectivamente: TCP/IP (uma única série), TCP/IP (várias séries), Sintético Multifractal, Sintético $\mathrm{fBm}$ e de Vídeo. As figuras mostram a quantidade de séries agregadas versus a probabilidade de perda. Podemos notar que os resultados obtidos pela proposta apresentam maior proximidade dos resultados de simulação com tráfego real do que para os demais métodos, para todos os tráfegos analisados. 
Apresentamos na Figura 2 a comparação de alguns dos métodos citados neste trabalho com a proposta feita para a série de tráfego dec-pkt-2 e observamos que o método proposto apresenta um resultado mais preciso do que as outras abordagem.

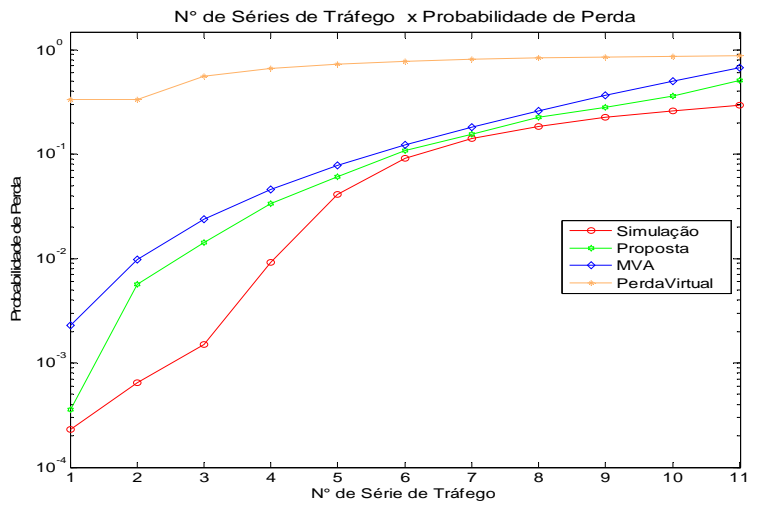

Fig 2. $\mathrm{N}^{\circ}$ de Séries de Tráfego versus Probabilidade de Perda para um Traço de Tráfego TCP/IP (dec-pkt-2)

Para a Figura 3 a análise foi feita para diversas séries de tráfego TCP/IP e observamos que o método proposto apresenta novamente uma melhor caracterização da perda de bytes com relação as abordagens consideradas.

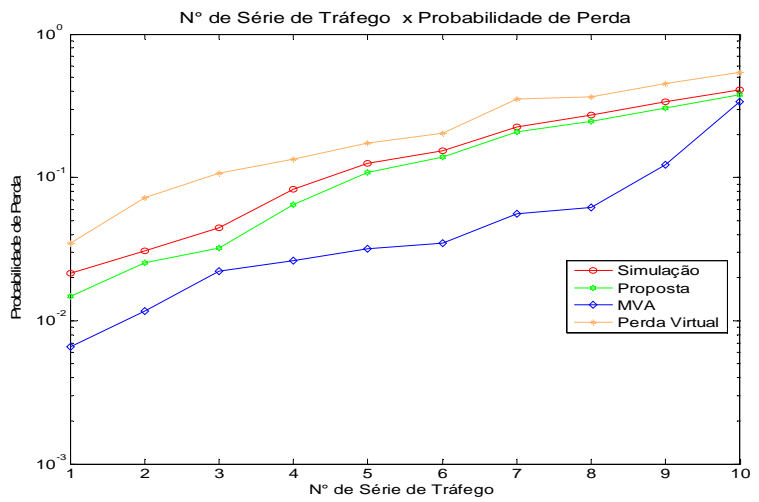

Fig 3. $\mathrm{N}^{\circ}$ de Séries de Tráfego versus Probabilidade de Perda TCP/IP Traço de Tráfego (Várias séries)

Na Figura 4 os resultados são mais expressivos, por modelar com certa exatidão um traço com características multifractais. De acordo com o resultado, pode-se concluir que o método MVA não seria uma boa estratégia para a caracterização para esta situação.

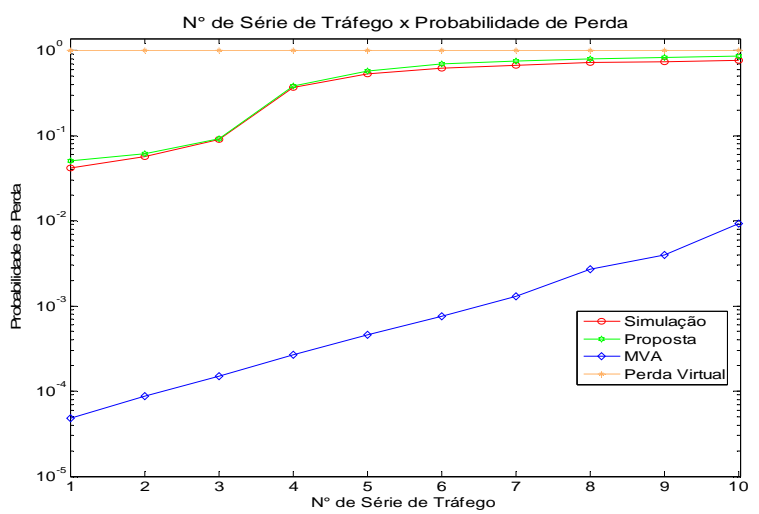

Fig 4. $\mathrm{N}^{\circ}$ de Séries de Tráfego versus Probabilidade de Perda para um Traço de Tráfego Sintético Multifractal
A Figura 5 apresenta os resultados de probabilidade de perda para séries (modelo fBm). Pela Figura 5, observa-se que mesmo as séries geradas pelo modelo $\mathrm{fBm}$ serem gaussianas e o método MVA ser baseado em tráfego gaussiano, o método proposto obteve resultados mais próximos aos das simulações.

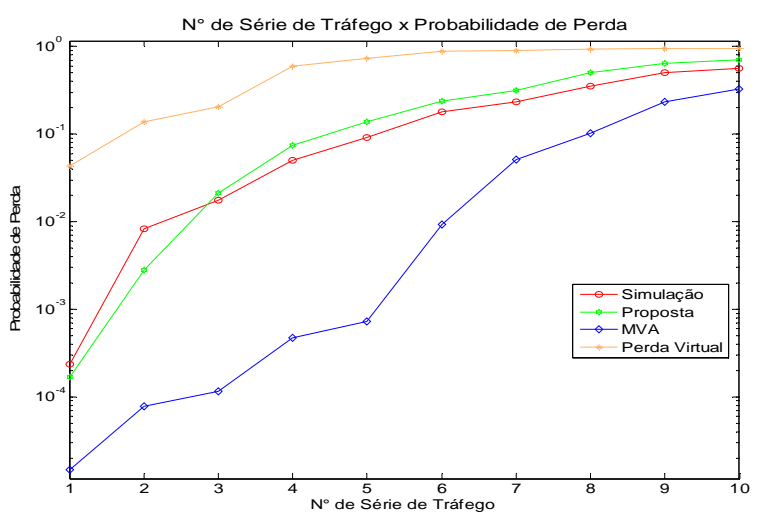

Fig 5. $\mathrm{N}^{\circ}$ de Séries de Tráfego versus Probabilidade de Perda para um Traço de Tráfego Sintético fBm

E por fim, observa-se na Figura 6 que o método proposto permanece mais preciso também para traços de vídeo.

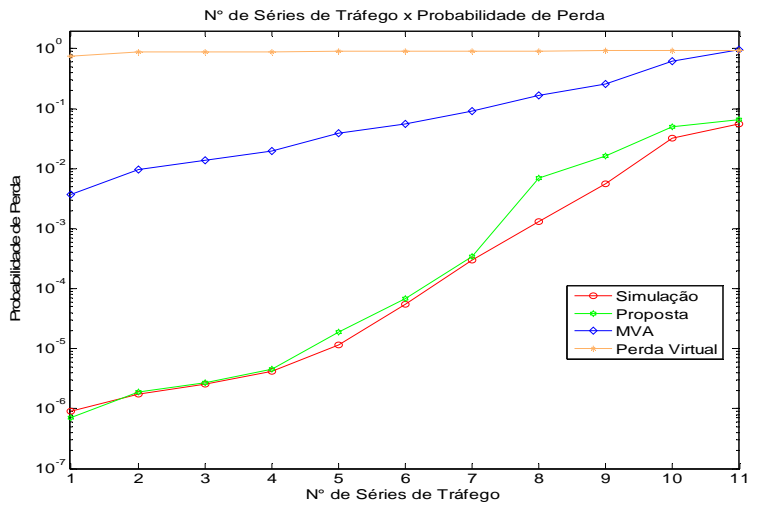

Fig 6. $\mathrm{N}^{\circ}$ de Séries de Tráfego versus Probabilidade de Perda para Traço de Tráfego de Vídeo

A Tabela II resume os resultados referentes à análise da quantidade de conexões aceitas assumindo várias probabilidades de perda referentes ao traço de vídeo (Aladdin, Diefirma, Diehard3, Dusk, FirstContact, Jurassic, RobinHood, Silence, Starqars4, Suzi and Strolch $e$ Trooper)[13].

TABELA II QUANTIDADE DE TRAÇOS DE TRÁFEGOS ACEITOS

\begin{tabular}{|c|c|c|c|c|}
\hline $\begin{array}{c}\text { Probabilidade de } \\
\text { Perda }\end{array}$ & Simulação & Proposta & MVA & $\begin{array}{c}\text { Perda } \\
\text { Virtual }\end{array}$ \\
\hline $10^{-4}$ & 6 & 6 & 0 & 0 \\
\hline $10^{-3}$ & 8 & 7 & 0 & 0 \\
\hline $10^{-2}$ & 9 & 9 & 2 & 0 \\
\hline
\end{tabular}

A Tabela II confirma que as quantidades de conexões aceitas pelo método proposto se equivalem ou estão bem próximas da simulação real. 


\section{CONCLUSÕES}

Em redes com suporte à qualidade de serviço, um algoritmo de controle de admissão (CAC - Connection Admission Control) determina se um novo fluxo de tráfego pode ser admitido na rede, tal que os usuários obtenham o desempenho de rede requerido.

Uma análise com tráfego multifractal pode se tornar complexa devido a algumas dificuldades, por exemplo, estimação de probabilidade de perda com fluxos apresentando densidade de probabilidade lognormal ou de cauda pesada [25]. Neste trabalho, derivamos uma equação de probabilidade de perda que nos possibilitou analisarmos a admissão de fluxos de tráfego considerando características multifractais. Apresentamos assim, um novo método de controle de admissão que se mostrou ser eficiente para vários tipos de tráfego reais de redes, sendo os mesmos multifractais ou não. Verificamos pelos resultados obtidos que o esquema de controle de admissão proposto é mais preciso em diferentes situações que vários métodos existentes na literatura. Dessa forma, o esquema de controle de admissão proposto pode ser empregado em diversas tecnologias de rede para garantir parâmetros de QoS requeridos aos fluxos de tráfego.

\section{REFERÊNCIAS:}

[1] Lazar, A., Pacifici, G., \& Pendarakis, D. (1994)." Modeling video sources for real time scheduling. ACM multimedia!" Syst.J., 1, 253-266.

[2] Knightly, E. \& Shroff, N. (1999). “Admission control for statistical QoS: Theory and practice”. IEEE Network, 13(2).

[3] S.B Jacobsen, K MOth, and L.Dittmann, "Load control in ATM networks," in Proc.8 th Int. Switch. Symp.,May 1990

[4] S.Sato and S Tanabe,"A study on ATM traffic burstiness,'IEICE tech, rep. IN88-142, Feb 1989.

[5] Shroff, N. \& Schwartz, M. (1998). "Improved loss calculations at an ATM multiplexer". Technical report, Sch. Elec. Comput. Eng., Purdue Univ., West Lafayette,.

[6] H Suzuki, T. Marase, S. Sato, and T. Takeuchi, “A simple and burst-variation independent measeure of service quality for ATM traffic control," in Proc. 7th ITC Sem.1990.

[7] Vinay J. Ribeiro, Rudolf H. Riedi, Matthew S. Crouse, and Richard G. Baraniuk."Multiscale Queuing Analysis of Long-Range-Dependent Network Traffic". IEEE INFOCOM 2000

[8] R. J. Gibbens and F. P. Kely, "Measurement-based connection admission control" 15 th International Teletraffic Congress Proceedings, Junho 1997.

[9]Baek-Young Choi, Zhenhai Duan, Zhi-Li Zhang, and David H.C. Du, "Measurement-based Admisson Control Using Wavelets for Broadband Networks"

[10] Sugih Jamin, Scott J. Shenker, Peter B. Danzig, "Comparison of Measurement-based Admission Control Algorithm for Crontrolled-Load Service".

[11] Sugih Jamin, Peter B. Danzig, Scott J. Shenker, and Lixia Zhang, "A Measurement-based Admission Control Agorithm for Integrated Services Packet Networks".

[12]Benes V.(1963) "General Stochastic Processes in the Theory of Queues Reading", MA: Addison Wesley.

[13] http://www.cs.columbia.edu/ hgs/internet/traces.html
[14] http://complex.futurs.inria.fr/FracLab/

[15] Riedi, R. H. et al.(1999) “A Multifractal Wavelet Model with Application to Network Traffic." IEEE Transactions on Information Theory, v.45, p.992-1018.

[16] T.Lee, K. Lai, And S. Duann, “ Design of a real-time call admission controller for Atm networks, " IEEE/ACM Transactions on Networking, vol.4,pp 758-765, Oct. 1996

[17] R.Loynes, "The Stability of a Queue with NonIndependent Inter-arrival and Service Times," Proc. Cambridge Philos. Soc.,vol.58, pp.497-520,1962.

[18] Kesidis, G., Walrand, J., \& Chang, C. S. (1993). "Effective bandwidths for multiclass markov fluids and other ATM sources." IEEE/ACM Trans. on Networking, 1(3), 424428.

[19] Elwalid, A. \& Mitra, D. (1993). "Effective bandwidth of general markovian traffic sources and admission control of high speed networks." IEEE/ACM Trans. Networking, 1(3).

[20] Chang, C. (1994). "Stability, queue length and delay of deterministic and stochastic queueing networks." IEEE Trans. Automat. Contr., 39, 913-931.

[21] Berger, A. W. \& Whitt, W. (1998). "Extending the effective bandwidth concept to networks with priority classes." IEEE Communications Magazine, 36(8), 78-84.

[22] Stenico, J. W. G., Vieira, F. H. T and Ling, LL " An approach for evaluating the buffer queueing behavior of multifractal network traffic flows, Proceedings IWT09 p. 114-119.

[23] MANDELBROT, B. B. "The fractal geometry of nature.” New York: W. H. Freeman and Company, 1982.

[24] Jens Milbrandt, Michael Menth, and Jan Junker "Experience -Based Admission Control in the Presence of Traffic Changes"

[25] Nelson X. Liu and John S Baras "Statistical Modeling and Performance Analysis of Multi-Scale Traffic", Proceedings of IEEE Infocom 2003, p.1837-1847.

[26] Ferrari, D. \& Verma, D. (1990). "A scheme for real-time channel establishment in wide-area networks." IEEE J. Select. Areas Commun., 8, 368-379.

[27]http://ita.ee.lbl.gov/html/traces.html

[28] Erramilli, A., Narayan, O., \& Willinger, W. (1996).

"Experimental queueing analysis with long-range dependent packet traffic".IEEE/ACM Trans. on Net., 4(2). 